\title{
Some Trapezium-Like Inequalities Involving Functions Having Strongly $n$-Polynomial Preinvexity Property of Higher Order
}

\author{
Muhammad Uzair Awan $\mathbb{D D}^{1},{ }^{1}$ Sadia Talib, ${ }^{1}$ Muhammad Aslam Noor, ${ }^{2}$ Yu-Ming Chu $\mathbb{D},{ }^{3,4}$ \\ and Khalida Inayat Noor ${ }^{2}$ \\ ${ }^{1}$ Department of Mathematics, Government College University, Faisalabad, Pakistan \\ ${ }^{2}$ Department of Mathematics, COMSATS University Islamabad, Islamabad, Pakistan \\ ${ }^{3}$ Department of Mathematics, Huzhou University, Huzhou 313000, China \\ ${ }^{4}$ Hunan Provincial Key Laboratory of Mathematical Modeling and Analysis in Engineering, Changsha University of Science \\ \& Technology, Changsha 410114, China
}

Correspondence should be addressed to Yu-Ming Chu; chuyuming@zjhu.edu.cn

Received 14 March 2020; Accepted 20 July 2020; Published 13 August 2020

Academic Editor: Syed Abdul Mohiuddine

Copyright (c) 2020 Muhammad Uzair Awan et al. This is an open access article distributed under the Creative Commons Attribution License, which permits unrestricted use, distribution, and reproduction in any medium, provided the original work is properly cited.

The main objective of this paper is to introduce a new class of preinvex functions which is called as $n$-polynomial preinvex functions of a higher order. As applications of this class of functions, we discuss several new variants of trapezium-like inequalities. In order to obtain the main results of the paper, we use the concepts and techniques of $k$-fractional calculus. We also discuss some special cases of the obtained results which show that the main results of the paper are quite unifying one.

\section{Introduction}

Convexity is one of the most important and natural notions in mathematics; it plays a significant role in various branches of pure and applied sciences [1-15]. For example, the set of feasible points in optimization theory is convex; the loss function used to measure the quality of solution in statistics is convex. In particular, many remarkable inequalities have been established via the convexity theory [16-32].

Recently, the classical concept of convexity has been extended and generalized in different directions. For instance, Hanson [33] introduced the notion of differentiable invex function but did not name it as invex; Craven [34] gave the term invex for this class of functions due to their property described as invariance by convexity. Mititelu [35] introduced the notion of invex set as follows.
Definition 1 (see [35]). Let $\mathscr{X} \in \mathbb{R}$ be a nonempty set and $\zeta: \mathscr{X} \times \mathscr{X} \mapsto \mathbb{R}$ be a real-valued function. Then $\mathscr{X}$ is said to be invex with respect to $\zeta$ if

$$
x+t \zeta(y, x) \in \mathscr{X}
$$

for all $x, y \in \mathscr{X}$ and $t \in[0,1]$.

We clearly see that the invexity reduces to the classical convexity if $\zeta(y, x)=y-x$. Therefore, every convex set is an invex with respect to $\zeta(y, x)=y-x$, but its converse is not true in general [35].

Weir and Mond [36] introduced the class of preinvex functions by use of the invex set.

Definition 2 (see [36]). Let $\mathscr{X} \in \mathbb{R}$ be a nonempty invex set with respect to $\zeta: \mathscr{X} \times \mathscr{X} \mapsto \mathbb{R}$. Then the function 
$\mathscr{F}: \mathscr{X} \mapsto \mathbb{R}$ is said to be preinvex with respect to $\zeta$ if the inequality

$$
\mathscr{F}(x+t \zeta(y, x)) \leq(1-t) \mathscr{F}(x)+t \mathscr{F}(y)
$$

holds for all $x, y \in \mathscr{X}$ and $t \in[0,1]$.

Note that the preinvex function becomes the classical convex function if $\zeta(y, x)=y-x$; for more details regarding recent study on preinvexity property, we recommend the literature [37].

Very recently, Toplu et al. [38] introduced and investigated a new class of convexity named $n$-polynomial convexity, and Karamardian [39] and Polyak [40] independently introduced the class of strongly convex functions. Strong convexity is the strengthening of convexity.

Definition 3 (see $[39,40]$ ). A function $\mathscr{F}: \mathscr{X} \mapsto \mathbb{R}$ is said to be strongly convex with respect to modulus $\mu>0$ if the inequality

$$
\mathscr{F}((1-t) x+t y) \leq(1-t) \mathscr{F}(x)+t F(y)-\mu t(1-t)(y-x)^{2}
$$

holds for all $x, y \in \mathscr{X}$ and $t \in[0,1]$.

Lin et al. [41] introduced higher order strongly convex functions to simplify the study of mathematical programs with equilibrium constraints.

Definition 4 (see [41]). Let $\sigma, \mu>0$. Then, the function $\mathscr{F}: \mathscr{X} \mapsto \mathbb{R}$ is said to be $\sigma$-order strongly convex with respect to modulus $\mu$ if

$$
\mathscr{F}((1-t) x+t y) \leq(1-t) \mathscr{F}(x)+t F(y)-\mu t(1-t)|y-x|^{\sigma}
$$

for all $x, y \in \mathscr{X}$ and $t \in[0,1]$.

If $\sigma=2$, then Definition 4 becomes Definition 3. Therefore, higher order strong convexity is a generalization of strong convexity. Lin et al. [41] proved that the higher order strong convexity of a function is equivalent to higher order strong monotonicity of the gradient map of the function.

It is well known that the Hermite-Hadamard inequality [42-45] is one of the most important and classical inequalities in convex function theory, which can be stated as follows.

Theorem 5. Let $\mathscr{F}:[a, b] \subset \mathbb{R} \mapsto \mathbb{R}$ be a convex function. Then

$$
\mathscr{F}\left(\frac{a+b}{2}\right) \leq \frac{1}{b-a} \int_{a}^{b} \mathscr{F}(x) d x \leq \frac{\mathscr{F}(a)+\mathscr{F}(b)}{2}
$$

In recent decades, the fractional calculus has become a powerful tool in numerous branches of mathematics, physics, and engineering. The history of fractional calculus dates back to 1695 with the work of mathematicians such as L'Hospital and Leibniz, but the logical definitions were proposed by Liouville in 1834, Riemann in 1847, and Grünwald in 1867. Fractional calculus can be considered a super set of integer- order calculus, which has the potential to accomplish what integer-order calculus cannot. The classical form of the fractional calculus is given by the Riemann-Liouville integrals as follows.

Definition 6 (see [46]). Let $\alpha>0,0 \leq a<b$, and $\mathscr{F} \in L_{1}[a, b]$. Then, the $\alpha$-order Riemann-Liouville integrals $\mathfrak{\Im}_{a^{+}}^{\alpha} \mathscr{F}$ and $\mathfrak{\Im}_{b^{-}}^{\alpha} \mathscr{F}$ are defined by

$$
\begin{aligned}
& \mathfrak{\mho}_{a^{+}}^{\alpha} \mathscr{F}(x)=\frac{1}{\Gamma(\alpha)} \int_{a}^{x}(x-t)^{\alpha-1} \mathscr{F}(t) \mathrm{d} t \quad(x>a), \\
& \mathfrak{\Im}_{b^{-}}^{\alpha} \mathscr{F}(x)=\frac{1}{\Gamma(\alpha)} \int_{x}^{b}(t-x)^{\alpha-1} \mathscr{F}(t) \mathrm{d} t \quad(x<b),
\end{aligned}
$$

respectively, where

$$
\Gamma(\alpha)=\int_{0}^{\infty} e^{-t} t^{\alpha-1} \mathrm{~d} t
$$

is the Euler gamma function.

Sarikaya et al. [47] used the fractional calculus to obtain new variants of the Hermite-Hadamard inequality, which opened a new research area for the people who are working on the field of mathematical inequalities, particularly working on the inequalities involving convexity and its generalizations. For some more recent research work done in this direction, see [48-50].

Diaz et al. [51] introduced the generalized $k$-gamma function $\Gamma_{k}(x)$ and $k$-beta function $B_{k}(x, y)$ as follows.

$$
\begin{aligned}
\Gamma_{k}(x) & =\int_{0}^{\infty} t^{x-1} e^{-t^{k} / k} \mathrm{~d} t \\
B_{k}(x, y) & =\frac{1}{k} \int_{0}^{1} t^{x / k-1}(1-t)^{\mathrm{y} / k-1} \mathrm{~d} t=\frac{\Gamma_{k}(x) \Gamma_{k}(y)}{\Gamma_{k}(x+y)} .
\end{aligned}
$$

Making use of the generalized $k$-gamma function, Sarikaya et al. [52] introduced the $k$-Riemann-Liouville fractional integrals and discussed their properties and applications.

Definition 7 (see [52]). Let $\alpha, k>0,0 \leq a<b$, and $\mathscr{F} \in L_{1}[a, b]$. Then, the $\alpha$-order $k$-Riemann-Liouville integrals ${ }_{k} \widetilde{J}_{a^{+}}^{\alpha} \mathscr{F}$ and ${ }_{k} \mathfrak{\Im}_{b^{-}}^{\alpha} \mathscr{F}$ are defined by

$$
\begin{aligned}
& { }_{k} \mathfrak{\mho}_{a^{+}}^{\alpha} \mathscr{F}(x)=\frac{1}{k \Gamma_{k}(\alpha)} \int_{a}^{x}(x-t)^{a / k-1} \mathscr{F}(t) \mathrm{d} t \quad(x>a), \\
& { }_{k} \widetilde{\mho}_{b^{-}}^{\alpha} \mathscr{F}(x)=\frac{1}{k \Gamma_{k}(\alpha)} \int_{x}^{b}(t-x)^{a / k-1} \mathscr{F}(t) \mathrm{d} t \quad(x<b) .
\end{aligned}
$$

In recent years, several authors have used the concepts of $k$-fractional calculus in obtaining new variants of fractional analogues of classical inequalities. For example, Huang et al. [53] obtained $k$-fractional conformable analogues of Hermite-Hadamard's inequality. Rahman et al. [54] obtained fractional analogues of the Gruss type inequalities using $k$ conformable fractional integral operators. 
The aim of the article is to obtain some new $k$-analogues of trapezium-like inequalities involving a new class of functions called strongly $n$-polynomial preinvex function of higher order. We also discuss some special cases of the obtained results. We expect that the ideas and techniques of this article will inspire interested readers working in this field. This is the main motivation of the article.

\section{Results and Discussions}

In this section, we discuss our main results. First of all, let us give the definition of strongly $n$-polynomial preinvex function of higher order.

Definition 8 . Let $n \in \mathbb{N}$ and $\mathscr{X} \in \mathbb{R}$ be a nonempty invex set with respect to $\zeta: \mathscr{X} \times \mathscr{X} \mapsto \mathbb{R}$. A nonnegative function $\mathscr{F}: \mathscr{X} \mapsto \mathbb{R}$ is said to be strongly $n$-polynomial preinvex function of higher order, if

$$
\begin{aligned}
\mathscr{F}(a & +t \zeta(b, a)) \\
\leq & \frac{1}{n} \sum_{i=1}^{n}\left[1-t^{i}\right] \mathscr{F}(a)+\frac{1}{n} \sum_{i=1}^{n}\left[1-(1-t)^{i}\right] \mathscr{F}(b) \\
& \quad-\mu\left[t^{\sigma}(1-t)+t(1-t)^{\sigma}\right]\|\zeta(b, a)\|^{\sigma},
\end{aligned}
$$

$\forall a, b \in \mathscr{X}, t \in[0,1], \mu, \sigma>0$,

Note that if $\sigma=2$, then the class of strongly $n$-polynomial preinvex function of higher order reduces to the class of strongly $n$-polynomial preinvex function which is also new class. If we consider $\mu=0$, then the class of strongly $n$-polynomial preinvex function of higher order reduces to the class of $n$-polynomial preinvex functions which is also new in the literature. Similarly, if we take $\zeta(b, a)=b-a$, then we have new class of strongly $n$-polynomial convex function of higher order. If we take $n=1$, then the above class reduces to simple strongly preinvex function of higher order. If we take $n=2$, then we have strongly 2-polynomial preinvex function of higher order:

$$
\begin{aligned}
\mathscr{F}(a+t \zeta(b, a)) \leq & \frac{2-t-t^{2}}{2} \mathscr{F}(a)+\frac{3 t-t^{2}}{2} \mathscr{F}(b) \\
& -\mu\left[t^{\sigma}(1-t)+t(1-t)^{\sigma}\right]\|\zeta(b, a)\|^{\sigma},
\end{aligned}
$$$$
\forall a, b \in \mathscr{X}, t \in[0,1], \mu, \sigma>0 \text {, }
$$

In order to obtain following result, we need the famous Condition $\mathrm{C}$ which was introduced by Mohan and Neogy [55].

Condition $C$. Let $\mathscr{X} \subset \mathbb{R}$ be an invex set with respect to bifunction $\zeta(\cdot, \cdot)$. Then, for any $x, y \in \mathscr{X}$ and $t \in[0,1]$,

$$
\begin{aligned}
& \zeta(y, y+t \zeta(x, y))=-t \zeta(x, y) \\
& \zeta(x, y+t \zeta(x, y))=(1-t) \zeta(x, y)
\end{aligned}
$$

Note that for every $x, y \in \mathscr{X}, t_{1}, t_{2} \in[0,1]$, and from Condition $\mathrm{C}$, we have

$$
\zeta\left(y+t_{2} \zeta(x, y), y+t_{1} \zeta(x, y)\right)=\left(t_{2}-t_{1}\right) \zeta(x, y)
$$

We now give our first main result.

Theorem 9. Let $\mathscr{F}:[a, a+\zeta(b, a)] \mapsto \mathbb{R}$ be an strongly $n$ polynomial preinvex function with $\zeta(b, a)>0$ and $\mathscr{F} \in L[a$, $a+\zeta(b, a)]$. If $\zeta(\cdot, \cdot)$ satisfies Condition $C$, then

$$
\begin{aligned}
& \left(\frac{n}{n+2^{-n}-1}\right)\left[\mathscr{F}\left(\frac{2 a+\zeta(b, a)}{2}\right)+\frac{\mu\left(\alpha^{2}-\alpha k+2 k\right)}{4(\alpha+k)(\alpha+2 k)}\|\zeta(b, a)\|^{\sigma}\right] \\
& \leq \frac{\Gamma_{k}(\alpha+k)}{\zeta^{\alpha / k}(b, a)}\left[{ }_{k} \mathfrak{\mho}_{a^{+}}^{\alpha} \mathscr{F}(a+\zeta(b, a))+{ }_{k} \mathfrak{\mho}_{[a+\zeta(b, a)]^{\alpha}}^{\alpha} \mathscr{F}(a)\right] \\
& \leq\left[\frac{\mathscr{F}(a)+\mathscr{F}(b)]}{n}\right] \sum_{i=1}^{n} \frac{2 s k}{\alpha+s k}-\frac{2 k \alpha \mu\|\zeta(b, a)\|^{\sigma}}{(\alpha+k)(\alpha+2 k)} .
\end{aligned}
$$

Proof. Since it is given that $\mathscr{F}$ is an an strongly $n$-polynomial preinvex function and $\zeta(\cdot, \cdot)$ satisfies Condition $C$, then

$$
\begin{aligned}
\mathscr{F}( & \left.\frac{2 a+\zeta(b, a)}{2}\right) \\
\leq & \frac{1}{n} \sum_{i=1}^{n}\left[1-\frac{1}{2^{i}}\right]\{\mathscr{F}(a+t \zeta(b, a))+\mathscr{F}(a+(1-t) \zeta(b, a))\} \\
& -\frac{\mu}{4} \zeta^{2}(a+(1-t) \zeta(b, a), a+t \zeta(b, a)) \\
= & \frac{1}{n} \sum_{i=1}^{n}\left[1-\frac{1}{2^{i}}\right]\{\mathscr{F}(a+t \zeta(b, a))+\mathscr{F}(a+(1-t) \zeta(b, a))\} \\
& -\frac{\mu}{4}(1-2 t)^{2} \zeta^{2}(b, a) .
\end{aligned}
$$

Multiplying the above inequality by $t^{\alpha / k-1}$ and then integrating the above inequality with respect to $t$ on $[0,1]$ yields

$$
\begin{aligned}
& \left(\frac{n}{n+2^{-n}-1}\right)\left[\frac{k}{\alpha} \mathscr{F}\left(\frac{2 a+\zeta(b, a)}{2}\right)+\frac{\mu k\left(\alpha^{2}-\alpha k+2 k\right)}{4 \alpha(\alpha+k)(\alpha+2 k)} \zeta^{2}(b, a)\right] \\
& \quad \leq \int_{0}^{1} t^{a / k-1} \mathscr{F}(a+t \zeta(b, a)) \mathrm{d} t+\int_{0}^{1} t^{a / k-1} \mathscr{F}(a+(1-t) \zeta(b, a)) \mathrm{d} t \\
& \quad=I_{1}+I_{2} .
\end{aligned}
$$

Now

$$
\begin{aligned}
I_{1} & =\int_{a}^{a+\zeta(b, a)}\left(\frac{u-a}{\zeta(b, a)}\right)^{a / k-1} \mathscr{F}(u) \frac{d u}{\zeta(b, a)} \\
& =\frac{k \Gamma_{k}(\alpha)}{\zeta^{\alpha / k}(b, a)} k \widetilde{\mho}_{[a+\zeta(b, a)]^{-}}^{\alpha} \mathscr{F}(a) .
\end{aligned}
$$

Similarly

$$
I_{2}=\frac{k \Gamma_{k}(\alpha)}{\zeta^{\alpha / k}(b, a)}{ }_{k} \mathfrak{\mho}_{a^{+}}^{\alpha} \mathscr{F}(a+\zeta(b, a)) .
$$


This imples

$$
\begin{gathered}
\left(\frac{n}{n+2^{-n}-1}\right)\left[\mathscr{F}\left(\frac{2 a+\zeta(b, a)}{2}\right)+\frac{\mu\left(\alpha^{2}-\alpha k+2 k\right)}{4(\alpha+k)(\alpha+2 k)} \zeta^{2}(b, a)\right] \\
\leq \frac{\Gamma_{k}(\alpha+k)}{\zeta^{\alpha / k}(b, a)}\left[{ }_{k} \mathfrak{\mho}_{a^{+}}^{\alpha} \mathscr{F}(a+\zeta(b, a))+{ }_{k} \mathfrak{\mho}_{[a+\zeta(b, a)]^{\alpha}}^{\alpha} \mathscr{F}(a)\right] .
\end{gathered}
$$

For the proof of right-hand side inequality,

$$
\begin{aligned}
\mathscr{F}(a+t \zeta(b, a))+\mathscr{F}(a+(1-t) \zeta(b, a)) \\
\leq[\mathscr{F}(a)+\mathscr{F}(b)]\left[\frac{1}{n} \sum_{i=1}^{n}\left[1-t^{i}\right]+\frac{1}{n} \sum_{i=1}^{n}\left[1-(1-t)^{i}\right]\right] \\
\quad-2 \mu t(1-t) \zeta^{2}(b, a) .
\end{aligned}
$$

Multiplying the above inequality by $t^{\alpha / k-1}$, then integrating the resulting inequality with respect to $t$ over $[0,1]$, we obtain

$$
\begin{aligned}
\frac{k \Gamma_{k}(\alpha)}{\zeta^{\alpha / k}(b, a)}\left[{ }_{k} \widetilde{\mho}_{a^{+}}^{\alpha} \mathscr{F}(a+\zeta(b, a))+{ }_{k} \widetilde{\mho}_{[a+\zeta(b, a)]^{-}}^{\alpha} \mathscr{F}(a)\right] \\
\leq\left[\frac{\mathscr{F}(a)+\mathscr{F}(b)}{n}\right] \sum_{i=1}^{n} \int_{0}^{1} t^{a / k-1}\left[2-t^{i}-(1-t)^{i}\right] \mathrm{d} t \\
\quad-\frac{2 k^{2} \mu \zeta^{2}(b, a)}{(\alpha+k)(\alpha+2 k)} .
\end{aligned}
$$

This implies that

$$
\begin{gathered}
\frac{\Gamma_{k}(\alpha+k)}{\zeta^{\alpha / k}(b, a)}\left[{ }_{k} \mathfrak{\mho}_{a^{+}}^{\alpha} \mathscr{F}(a+\zeta(b, a))+{ }_{k} \mathfrak{\mho}_{[a+\zeta(b, a)]^{\alpha}}^{\alpha} \mathscr{F}(a)\right] \\
\leq\left[\frac{\mathscr{F}(a)+\mathscr{F}(b)]}{n}\right] \sum_{i=1}^{n} \frac{2 s k}{\alpha+s k}-\frac{2 k \alpha \mu \zeta^{2}(b, a)}{(\alpha+k)(\alpha+2 k)} .
\end{gathered}
$$

Combining (19) and (22) completes the proof.

Note that if we take $\alpha=k=n=1$ in Theorem 9, then we get the Hermite-Hadamard like inequality involving strongly preinvex functions.

We now derive a new auxiliary result which will be helpful in obtaining the next results of the article.

Lemma 10. Let $\mathscr{F}:[a, a+\zeta(b, a)] \mapsto \mathbb{R}$ be a differentiable function and $\mathscr{F}^{\prime} \in[a, a+\zeta(b, a)]$. Then, for any $0<\alpha \leq 1$, $0<\lambda \leq 1$, the following equality for $k$-fractional integrals holds:

$$
\begin{aligned}
& \frac{(k+\alpha(1-\lambda)) \mathscr{F}(a+\zeta(b, a))+(k-\alpha(1-\lambda)) \mathscr{F}(a)}{2 k} \\
& \quad-\frac{\Gamma_{k}(\alpha+k)}{2 \zeta^{\alpha / k}(b, a)}\left({ }_{k} \mathfrak{\mho}_{a^{+}}^{\alpha} \mathscr{F}(a+\zeta(b, a))+{ }_{k} \widetilde{\mho}_{(a+\zeta(b, a))^{-}}^{\alpha} \mathscr{F}(a)\right) \\
& =\frac{\zeta(b, a)}{2} \int_{0}^{1}\left(t^{a / k}+\frac{\alpha}{k}(1-\lambda)-(1-t)^{a / k}\right) \mathscr{F}^{\prime}(a+t \zeta(b, a)) d t .
\end{aligned}
$$

Proof. It suffices to show that

$$
\begin{aligned}
J= & \frac{\zeta(b, a)}{2}\left[\int_{0}^{1}\left(t^{a / k}+\frac{\alpha}{k}(1-\lambda)-(1-t)^{a / k}\right) \mathscr{F}^{\prime}(a+t \zeta(b, a)) \mathrm{d} t\right] \\
= & \frac{\zeta(b, a)}{2}\left[\int_{0}^{1}\left(t^{a / k}+\frac{\alpha}{k}(1-\lambda)\right) \mathscr{F}^{\prime}(a+t \zeta(b, a)) \mathrm{d} t\right. \\
& \left.-\int_{0}^{1}(1-t)^{a / k} \mathscr{F}^{\prime}(a+t \zeta(b, a)) \mathrm{d} t\right]=\frac{\zeta(b, a)}{2}\left[J_{1}+J_{2}\right] .
\end{aligned}
$$

Integrating by parts

$$
\begin{aligned}
J_{1}= & \int_{0}^{1}\left(t^{a / k}+\frac{\alpha}{k}(1-\lambda)\right) \mathscr{F}^{\prime}(a+t \zeta(b, a)) \mathrm{d} t \\
= & \frac{(\alpha(1-\lambda)+k) \mathscr{F}(a+\zeta(b, a))-\alpha(1-\lambda) \mathscr{F}(a)}{k \zeta(b, a)} \\
& -\frac{\alpha}{k \zeta(b, a)} \int_{0}^{1} t^{a / k} \mathscr{F}(a+t \zeta(b, a)) \mathrm{d} t \\
= & \frac{(\alpha(1-\lambda)+k) \mathscr{F}(a+\zeta(b, a))-\alpha(1-\lambda) \mathscr{F}(a)}{k \zeta(b, a)} \\
& -\frac{\alpha}{k \zeta^{(\alpha / k)+1}(b, a)} \int_{a}^{a+\zeta(b, a)}(u-a)^{a / k-1} \mathscr{F}(u) \mathrm{d} u \\
= & \frac{(\alpha(1-\lambda)+k) \mathscr{F}(a+\zeta(b, a))-\alpha(1-\lambda) \mathscr{F}(a)}{k \zeta(b, a)} \\
& \left.-\frac{\Gamma_{k}(\alpha+k)}{\zeta^{a / k+1}(b, a)_{k}} \widetilde{J}_{(a+\zeta(b, a))^{-}}^{\alpha} \mathscr{F}(a)\right) .
\end{aligned}
$$

Similarly,

$$
\begin{aligned}
J_{2} & =-\int_{0}^{1}(1-t)^{a / k} \mathscr{F}^{\prime}(a+t \zeta(b, a)) \mathrm{d} t \\
& =\frac{\mathscr{F}(a)}{\zeta(b, a)}-\frac{\alpha}{k \zeta(b, a)} \int_{0}^{1}(1-t)^{a / k} \mathscr{F}(a+t \zeta(b, a)) \mathrm{d} t \\
& =\frac{\mathscr{F}(a)}{\zeta(b, a)}-\frac{\alpha}{k \zeta^{a / k+1}(b, a)} \int_{a}^{a+\zeta(b, a)}(a+\zeta(b, a)-u)^{a / k-1} \mathscr{F}(u) \mathrm{d} u \\
& \left.=\frac{\mathscr{F}(a)}{\zeta(b, a)}-\frac{\Gamma_{k}(\alpha+k)}{\zeta^{a / k+1}(b, a)_{k}} \mathfrak{\Im}_{(a)^{+}}^{\alpha} \mathscr{F}(a+\zeta(b, a))\right) .
\end{aligned}
$$

Using (25) and (26) in (24) completes the proof. 
Theorem 11. Let $\mathscr{F}:[a, a+\zeta(b, a)] \mapsto \mathbb{R}$ be a differentiable function on $(a, a+\zeta(b, a))$ with $\zeta(b, a)>0$ and $\mathscr{F}^{\prime} \in L[a$, $a+\zeta(b, a)]$. If $\left|\mathscr{F}^{\prime}\right|$ is a strongly n-polynomial preinvex function of higher order, then

$$
\begin{aligned}
& \mid \frac{(k+\alpha(1-\lambda)) \mathscr{F}(a+\zeta(b, a))+(k-\alpha(1-\lambda)) \mathscr{F}(a)}{2 k} \\
& \quad-\frac{\Gamma_{k}(\alpha+k)}{2 \zeta^{\alpha / k}(b, a)}\left({ }_{k} \widetilde{\mho}_{a^{+}}^{\alpha} \mathscr{F}(a+\zeta(b, a))+{ }_{k} \widetilde{\mho}_{(a+\zeta(b, a))^{-}}^{\alpha} \mathscr{F}(a)\right) \mid \\
& \leq \frac{\zeta(b, a)}{2 n}\left[\left|\mathscr{F}^{\prime}(a)\right| \sum_{i=1}^{n} \mathscr{M}_{1}+\left|\mathscr{F}^{\prime}(b)\right| \sum_{i=1}^{n} \mathscr{M}_{2}-\mu\|\zeta(b, a)\|^{\sigma} \mathscr{M}_{3}\right],
\end{aligned}
$$

where

$$
\begin{aligned}
\mathscr{M}_{1}= & \frac{\alpha i(1-\lambda)}{k(i+1)}+k B_{k}(\alpha+k, s k+k)-\frac{k}{\alpha+s k+k}, \\
\mathscr{M}_{2}= & \frac{k}{\alpha+s k+k}-k B_{k}(s k+k, \alpha+k)+\frac{i \alpha(1-\lambda)}{k(i+1)}, \\
\mathscr{M}_{3}= & \frac{2 \alpha(1-\lambda)}{k(\sigma+1)(\sigma+2)}-k B_{k}(\sigma k+k, \alpha+k) \\
& +k B_{k}(\alpha+2 k, \sigma k+k)+k B_{k}(\sigma k+2 k, \alpha+k) .
\end{aligned}
$$

Proof. Using Lemma 10 and the fact that $\left|\mathscr{F}^{\prime}\right|$ is a strongly $n$ polynomial preinvex function of higher order, we have

$\mid(k+\alpha(1-\lambda)) \mathscr{F}(a+\zeta(b, a))+(k-\alpha(1-\lambda)) \mathscr{F}(a)$

$$
\begin{aligned}
& -\frac{\Gamma_{k}(\alpha+k)}{2 \zeta^{a / k}(b, a)}\left({ }_{k} \widetilde{J}_{a^{+}}^{\alpha} \mathscr{F}(a+\zeta(b, a))+{ }_{k} \mathfrak{J}_{(a+\zeta(b, a))^{-}}^{\alpha} \mathscr{F}(a)\right) \mid \\
= & \frac{\zeta(b, a)}{2}\left|\int_{0}^{1}\left(t^{a / k}+\frac{\alpha}{k}(1-\lambda)-(1-t)^{a / k}\right) \mathscr{F}^{\prime}(a+t \zeta(b, a)) \mathrm{d} t\right| \\
\leq & \frac{\zeta(b, a)}{2} \int_{0}^{1}\left(t^{a / k}+\frac{\alpha}{k}(1-\lambda)-(1-t)^{a / k}\right)\left|\mathscr{F}^{\prime}(a+t \zeta(b, a))\right| \mathrm{d} t \\
\leq & \frac{\zeta(b, a)}{2} \int_{0}^{1}\left(t^{a / k}+\frac{\alpha}{k}(1-\lambda)-(1-t)^{a / k}\right)\left[\frac{1}{n} \sum_{i=1}^{n}\left[1-t^{i}\right]\left|\mathscr{F}^{\prime}(a)\right|\right. \\
& \left.+\frac{1}{n} \sum_{i=1}^{n}\left[1-(1-t)^{i}\right]\left|\mathscr{F}^{\prime}(b)\right|-\mu \|\left.\zeta(b, a)\right|^{\sigma}\left[t^{\sigma}(1-t)+t(1-t)^{\sigma}\right]\right] \mathrm{d} t \\
= & \frac{\zeta(b, a)}{2 n}\left[\left|\mathscr{F}^{\prime}(a)\right| \int_{0}^{1}\left(t^{a / k}+\frac{\alpha}{k}(1-\lambda)-(1-t)^{a / k}\right) \sum_{i=1}^{n}\left[1-t^{i}\right] \mathrm{d} t\right. \\
& +\left|\mathscr{F}^{\prime}(b)\right| \int_{0}^{1}\left(t^{a / k}+\frac{\alpha}{k}(1-\lambda)-(1-t)^{a / k}\right) \sum_{i=1}^{n}\left[1-(1-t)^{i}\right] \mathrm{d} t \\
& \left.-\mu\|\zeta(b, a)\|^{\sigma} \int_{0}^{1}\left(t^{a / k}+\frac{\alpha}{k}(1-\lambda)-(1-t)^{a / k}\right)\left[t^{\sigma}(1-t)+t(1-t)^{\sigma}\right] \mathrm{d} t\right] \\
= & \frac{\zeta(b, a)}{2 n}\left[\left|\mathscr{F}^{\prime}(a)\right| \sum_{i=1}^{n} \int_{0}^{1}\left(t^{a / k}+\frac{\alpha}{k}(1-\lambda)-(1-t)^{a / k}\right)\left[1-t^{i}\right] \mathrm{d} t\right. \\
& +\left|\mathscr{F}^{\prime}(b)\right| \sum_{i=1}^{n} \int_{0}^{1}\left(t^{a / k}+\frac{\alpha}{k}(1-\lambda)-(1-t)^{a / k}\right)\left[1-(1-t)^{i}\right] \mathrm{d} t
\end{aligned}
$$

$$
\begin{aligned}
& \left.-\mu\|\zeta(b, a)\|^{\sigma} \int_{0}^{1}\left(t^{a / k}+\frac{\alpha}{\bar{k}}(1-\lambda)-(1-t)^{a / k}\right)\left[t^{\sigma}(1-t)+t(1-t)^{\sigma}\right] \mathrm{d} t\right] \\
= & \frac{\zeta(b, a)}{2 n}\left[\left|\mathscr{F}^{\prime}(a)\right| \sum_{i=1}^{n} \mathscr{M}_{1}+\left|\mathscr{F}^{\prime}(b)\right| \sum_{i=1}^{n} \mathscr{M}_{2}-\mu\|\zeta(b, a)\|^{\sigma} \mathscr{M}_{3}\right] .
\end{aligned}
$$

This completes the proof.

Theorem 12. Let $\mathscr{F}:[a, a+\zeta(b, a)] \mapsto \mathbb{R}$ be a differentiable function on $(a, a+\zeta(b, a))$ with $\zeta(b, a)>0, p^{-1}+q^{-1}=1$ and $\mathscr{F}^{\prime} \in L[a, a+\zeta(b, a)]$. If $\left|\mathscr{F}^{\prime}\right|^{q}$ is strongly $n$-polynomial preinvex function of higher order, then

$$
\begin{aligned}
& \mid \frac{(k+\alpha(1-\lambda)) \mathscr{F}(a+\zeta(b, a))+(k-\alpha(1-\lambda)) \mathscr{F}(a)}{2 k} \\
& \quad-\frac{\Gamma_{k}(\alpha+k)}{2 \zeta^{a / k}(b, a)}\left({ }_{k} \mathfrak{J}_{a^{+}}^{\alpha} \mathscr{F}(a+\zeta(b, a))+{ }_{k} \mathfrak{J}_{(a+\zeta(b, a))^{-}}^{\alpha} \mathscr{F}(a)\right) \mid \\
& \leq \frac{\zeta(b, a)}{2} \mathscr{M}_{4}\left(\frac{2}{n} \sum_{i=1}^{n} \frac{i}{i+1} A\left(\left|\mathscr{F}^{\prime}(a)\right|^{q},\left|\mathscr{F}^{\prime}(b)\right|^{q}\right)\right. \\
& \left.\quad-\frac{2 \mu\|\zeta(b, a)\|^{\sigma}}{(\sigma+1)(\sigma+2)}\right)^{1 / q},
\end{aligned}
$$

where

$$
\mathscr{M}_{4}=\frac{\alpha(1-\lambda)}{k}+2\left(\frac{k}{\alpha p+k}\right)^{1 / p}
$$

and $A(\cdot, \cdot)$ is the arithmetic mean.

Proof. Using Lemma 10, Hölder's integral inequality and $\left|\mathscr{F}^{\prime}\right|^{q}$ is strongly $n$-polynomial preinvex function of higher order, we have

$$
\begin{aligned}
& \mid \frac{(k+\alpha(1-\lambda)) \mathscr{F}(a+\zeta(b, a))+(k-\alpha(1-\lambda)) \mathscr{F}(a)}{2 k} \\
& \quad-\frac{\Gamma_{k}(\alpha+k)}{2 \zeta^{a / k}(b, a)}\left({ }_{k} \mathfrak{J}_{a^{+}}^{\alpha} \mathscr{F}(a+\zeta(b, a))+{ }_{k} \mathfrak{J}_{(a+\zeta(b, a))^{-}}^{\alpha} \mathscr{F}(a)\right) \mid \\
& =\frac{\zeta(b, a)}{2}\left|\int_{0}^{1}\left(t^{a / k}+\frac{\alpha}{k}(1-\lambda)-(1-t)^{a / k}\right) \mathscr{F}^{\prime}(a+t \zeta(b, a)) \mathrm{d} t\right| \\
& =\frac{\zeta(b, a)}{2}\left(\int_{0}^{1}\left(t^{a / k}+\frac{\alpha}{k}(1-\lambda)\right)\left|\mathscr{F}^{\prime}(a+t \zeta(b, a))\right| \mathrm{d} t\right. \\
& \left.\quad+\int_{0}^{1}(1-t)^{a / k}\left|\mathscr{F}^{\prime}(a+t \zeta(b, a))\right| \mathrm{d} t\right) \\
& \leq \frac{\zeta(b, a)}{2}\left[\left(\int_{0}^{1}\left(t^{a / k}+\frac{\alpha}{k}(1-\lambda)\right)^{p} \mathrm{~d} t\right)^{1 / p}\left(\int_{0}^{1}\left|\mathscr{F}^{\prime}(a+t \zeta(b, a))\right|^{q} \mathrm{~d} t\right)^{1 / q}\right. \\
& \left.\quad+\left(\int_{0}^{1}(1-t)^{a p / k} \mathrm{~d} t\right)^{1 / p}\left(\int_{0}^{1}\left|\mathscr{F}^{\prime}(a+t \zeta(b, a))\right|^{q} \mathrm{~d} t\right)^{1 / q}\right]
\end{aligned}
$$




$$
\begin{aligned}
\leq & \frac{\zeta(b, a)}{2}\left[\left(\int_{0}^{1} t^{a p / k} \mathrm{~d} t\right)^{1 / p}+\left(\int_{0}^{1}+\frac{\alpha^{p}}{k^{p}}(1-\lambda)^{p} \mathrm{~d} t\right)^{1 / p}\right. \\
& \left.+\left(\int_{0}^{1}(1-t)^{a p / k} \mathrm{~d} t\right)^{1 / p}\right] \times\left[\frac{\left|\mathscr{F}^{\prime}(a)\right|^{q}}{n} \sum_{i=1}^{n} \int_{0}^{1}\left[1-t^{i}\right] \mathrm{d} t\right. \\
& \left.+\frac{\left|\mathscr{F}^{\prime}(b)\right|^{q}}{n} \sum_{i=1}^{n} \int_{0}^{1}\left[1-(1-t)^{i}\right] \mathrm{d} t-\mu\|\zeta(b, a)\|^{\sigma} \int_{0}^{1}\left[t^{\sigma}(1-t)+t(1-t)^{\sigma}\right] \mathrm{d} t\right] \\
= & \frac{\zeta(b, a)}{2} \mathscr{M}_{4}\left[\frac{\left|\mathscr{F}^{\prime}(a)\right|^{q}}{n} \sum_{i=1}^{n} \frac{i}{i+1}+\frac{\left|\mathscr{F}^{\prime}(b)\right|^{q}}{n} \sum_{i=1}^{n} \frac{i}{i+1}-\frac{2 \mu\|\zeta(b, a)\|^{\sigma}}{(\sigma+1)(\sigma+2)}\right] \\
= & \frac{\zeta(b, a)}{2} \mathscr{M}_{4}\left(\frac{2}{n} \sum_{i=1}^{n} \frac{i}{i+1} A\left(\left|\mathscr{F}^{\prime}(a)\right|^{q},\left|\mathscr{F}^{\prime}(b)\right|^{q}\right)-\frac{2 \mu\|\zeta(b, a)\|^{\sigma}}{(\sigma+1)(\sigma+2)}\right)^{1 / q} .
\end{aligned}
$$

This completes the proof.

Theorem 13. Let $\mathscr{F}:[a, a+\zeta(b, a)] \mapsto \mathbb{R}$ be a differentiable function on $(a, a+\zeta(b, a))$ with $\zeta(b, a)>0, q>1$ and $\mathscr{F}^{\prime} \in$ $L[a, a+\zeta(b, a)]$. If $\left|\mathscr{F}^{\prime}\right|^{q}$ is strongly $n$-polynomial preinvex function of higher order, then

$$
\begin{aligned}
& \mid \frac{(k+\alpha(1-\lambda)) \mathscr{F}(a+\zeta(b, a))+(k-\alpha(1-\lambda)) \mathscr{F}(a)}{2 k} \\
& \quad-\frac{\Gamma_{k}(\alpha+k)}{2 \zeta^{\alpha / k}(b, a)}\left({ }_{k} \widetilde{\mho}_{a^{+}}^{\alpha} \mathscr{F}(a+\zeta(b, a))+{ }_{k} \widetilde{J}_{(a+\zeta(b, a))^{-}}^{\alpha} \mathscr{F}(a)\right) \mid \\
& \leq \frac{\zeta(b, a)}{2}\left(\frac{\alpha(1-\lambda)}{k}\right)^{1-1 / q}\left[\frac{\left|\mathscr{F}^{\prime}(a)\right|^{q}}{n} \sum_{i=1}^{n} \mathscr{M}_{1}\right. \\
& \left.\quad+\frac{\left|\mathscr{F}^{\prime}(b)\right|^{q}}{n} \sum_{i=1}^{n} \mathscr{M}_{2}-\mu \|\left.\zeta(b, a)\right|^{\sigma} \mathscr{M}_{3}\right],
\end{aligned}
$$

where $\mathscr{M}_{1}, \mathscr{M}_{2}$, and $\mathscr{M}_{3}$ are given in Theorem 11 .

Proof. Using Lemma 10, the power mean integral inequality and $\left|\mathscr{F}^{\prime}\right|^{q}$ is strongly $n$-polynomial preinvex function of higher order, we have

$$
\begin{aligned}
\mid \frac{(k+}{\alpha(1-\lambda)) \mathscr{F}(a+\zeta(b, a))+(k-\alpha(1-\lambda)) \mathscr{F}(a)} & 2 k \\
& -\frac{\Gamma_{k}(\alpha+k)}{2 \zeta^{\alpha / k}(b, a)}\left({ }_{k} \widetilde{J}_{a^{+}}^{\alpha} \mathscr{F}(a+\zeta(b, a))+{ }_{k} \widetilde{J}_{(a+\zeta(b, a))^{-}}^{\alpha} \mathscr{F}(a)\right) \mid \\
= & \frac{\zeta(b, a)}{2}\left|\int_{0}^{1}\left(t^{a / k}+\frac{\alpha}{k}(1-\lambda)-(1-t)^{a / k}\right) \mathscr{F}^{\prime}(a+t \zeta(b, a)) \mathrm{d} t\right| \\
\leq & \frac{\zeta(b, a)}{2} \int_{0}^{1}\left(t^{a / k}+\frac{\alpha}{k}(1-\lambda)-(1-t)^{a / k}\right)\left|\mathscr{F}^{\prime}(a+t \zeta(b, a))\right| \mathrm{d} t \\
\leq & \frac{\zeta(b, a)}{2}\left(\int_{0}^{1}\left(t^{a / k}+\frac{\alpha}{k}(1-\lambda)-(1-t)^{a / k}\right) \mathrm{d} t\right)^{1-1 / q} \\
\quad & \left(\int _ { 0 } ^ { 1 } ( t ^ { a / k } + \frac { \alpha } { k } ( 1 - \lambda ) - ( 1 - t ) ^ { a / k } ) \left[\frac{1}{n} \sum_{i=1}^{n}\left[1-t^{i}\right]\left|\mathscr{F}^{\prime}(a)\right|^{q}\right.\right. \\
& \left.\left.+\frac{1}{n} \sum_{i=1}^{n}\left[1-(1-t)^{i}\right]\left|\mathscr{F}^{\prime}(b)\right|^{q}-\mu\|\zeta(b, a)\|^{\sigma}\left[t^{\sigma}(1-t)+t(1-t)^{\sigma}\right]\right] \mathrm{d} t\right)^{1 / q}
\end{aligned}
$$

$$
\begin{aligned}
= & \frac{\zeta(b, a)}{2}\left(\frac{\alpha(1-\lambda)}{k}\right)^{1-1 / q}\left[\frac { | \mathscr { F } ^ { \prime } ( a ) | ^ { q } } { n } \int _ { 0 } ^ { 1 } \left(t^{a / k}+\frac{\alpha}{k}(1-\lambda)\right.\right. \\
& \left.-(1-t)^{a / k}\right) \sum_{i=1}^{n}\left[1-t^{i}\right] \mathrm{d} t+\frac{\left|\mathscr{F}^{\prime}(b)\right|^{q}}{n} \int_{0}^{1}\left(t^{a / k}+\frac{\alpha}{k}(1-\lambda)\right. \\
& \left.-(1-t)^{a / k}\right) \sum_{i=1}^{n}\left[1-(1-t)^{i}\right] \mathrm{d} t-\mu\|\zeta(b, a)\|^{\sigma} \int_{0}^{1}\left(t^{a / k}+\frac{\alpha}{k}(1-\lambda)\right. \\
& \left.\left.-(1-t)^{a / k}\right)\left[t^{\sigma}(1-t)+t(1-t)^{\sigma}\right] \mathrm{d} t\right]^{1 / q} \\
= & \frac{\zeta(b, a)}{2}\left(\frac{\alpha(1-\lambda)}{k}\right)^{1-1 / q}\left[\frac { | \mathscr { F } ^ { \prime } ( a ) | ^ { q } } { n } \sum _ { i = 1 } ^ { n } \int _ { 0 } ^ { 1 } \left(t^{a / k}+\frac{\alpha}{k}(1-\lambda)\right.\right. \\
& \left.-(1-t)^{a / k}\right)\left[1-t^{i}\right] \mathrm{d} t+\frac{\left|\mathscr{F}^{\prime}(b)\right|^{q}}{n} \sum_{i=1}^{n} \int_{0}^{1}\left(t^{a / k}+\frac{\alpha}{k}(1-\lambda)\right. \\
& \left.-(1-t)^{a / k}\right)\left[1-(1-t)^{i}\right] \mathrm{d} t-\mu\|\zeta(b, a)\|^{\sigma} \int_{0}^{1}\left(t^{a / k}+\frac{\alpha}{k}(1-\lambda)\right. \\
& \left.\left.-(1-t)^{a / k}\right)\left[t^{\sigma}(1-t)+t(1-t)^{\sigma}\right] \mathrm{d} t\right]^{1 / q} \\
= & \frac{\zeta(b, a)}{2}\left(\frac{\alpha(1-\lambda)}{k}\right)^{1-a / q}\left[\frac{\left|\mathscr{F}^{\prime}(a)\right|^{q}}{n} \sum_{i=1}^{n} \mathscr{M}_{1}+\frac{\left|\mathscr{F}^{\prime}(b)\right|^{q}}{n} \sum_{i=1}^{n} \mathscr{M}_{2}\right. \\
& \left.-\mu\|\zeta(b, a)\|^{\sigma} \mathscr{M}_{3}\right]^{1 / q} \cdot
\end{aligned}
$$

This completes the proof.

Theorem 14. Let $\mathscr{F}:[a, a+\zeta(b, a)] \mapsto \mathbb{R}$ be a differentiable function on $(a, a+\zeta(b, a))$ with $\zeta(b, a)>0, q>1$, and $\mathscr{F}^{\prime} \in$ $L[a, a+\zeta(b, a)]$. If $\left|\mathscr{F}^{\prime}\right|^{q}$ is strongly $n$-polynomial preinvex function of higher order, then

$$
\begin{aligned}
& \mid \frac{(k+\alpha(1-\lambda)) \mathscr{F}(a+\zeta(b, a))+(k-\alpha(1-\lambda)) \mathscr{F}(a)}{2 k} \\
& \quad-\frac{\Gamma_{k}(\alpha+k)}{2 \zeta^{\alpha / k}(b, a)}\left({ }_{k} \mathfrak{J}_{a^{+}}^{\alpha} \mathscr{F}(a+\zeta(b, a))+{ }_{k} \mathfrak{J}_{(a+\zeta(b, a))^{-}}^{\alpha} \mathscr{F}(a)\right) \mid \\
& \leq \frac{\zeta(b, a)}{2}\left(\frac{\alpha}{2 k}(1-\lambda)-\frac{\alpha k}{(\alpha+k)(\alpha+2 k)}\right)^{1-1 / q}\left(\frac{\left|\mathscr{F}^{\prime}(a)\right|^{q}}{n} \sum_{i=1}^{n} \mathscr{M}_{5}\right. \\
& \left.+\frac{\left|\mathscr{F}^{\prime}(b)\right|^{q}}{n} \sum_{i=1}^{n} \mathscr{M}_{6}-\mu \|\left.\zeta(b, a)\right|^{\sigma} \mathscr{M}_{7}\right)^{1 / q} \\
& +\frac{\zeta(b, a)}{2}\left(\frac{\alpha k}{(\alpha+k)(\alpha+2 k)}+\frac{\alpha}{2 k}(1-\lambda)\right)^{1-1 / q} \\
& \quad \cdot\left(\frac{\left|\mathscr{F}^{\prime}(a)\right|^{q}}{n} \sum_{i=1}^{n} \mathscr{M}_{8}+\frac{\left|\mathscr{F}^{\prime}(b)\right|^{q}}{n} \sum_{i=1}^{n} \mathscr{M}_{9}-\mu\|\zeta(b, a)\|^{\sigma} \mathscr{M}_{10}\right)^{1 / q}
\end{aligned}
$$

where

$$
\begin{aligned}
\mathscr{M}_{5}= & \frac{i \alpha(1-\lambda)(i+3)}{2 k(i+1)(i+2)}-k B_{k}(s k+2 k, \alpha+k)+k B_{k}(s k+k, \alpha+k) \\
& -\frac{k^{2}}{(\alpha+s k+k)(\alpha+s k+2 k)}-\frac{\alpha k}{(\alpha+k)(\alpha+2 k)},
\end{aligned}
$$




$$
\begin{aligned}
& \mathscr{M}_{6}=\frac{i \alpha(1-\lambda)}{2 k(i+2)}-k B_{k}(\alpha+k, s k+k)+k B_{k}(\alpha+2 k, s k+k) \\
& +\frac{k}{\alpha+s k+2 k} \\
& \mathscr{M}_{7}=\frac{2 k^{3}}{(k \sigma+\alpha+k)(k \sigma+\alpha+2 k)(k \sigma+\alpha+3 k)} \\
& +\frac{\alpha}{k}(1-\lambda)\left[\frac{2}{(\sigma+1)(\sigma+2)(\sigma+3)}+k B_{k}(2 k, k \sigma+k)\right. \\
& \left.-k B_{k}(\alpha+2 k, k \sigma+k)\right]+2 k B_{k}(k \sigma+2 k, \alpha+k) \\
& -k B_{k}(k \sigma+k, \alpha+k)+k B_{k}(\alpha+2 k, k \sigma+k) \\
& -k B_{k}(2 k, k \sigma+\alpha+k)-k B_{k}(k \sigma+3 k, \alpha+k) \\
& -k B_{k}(\alpha+3 k, k \sigma+k)+k B_{k}(3 k, k \sigma+\alpha+k) \text {, } \\
& \mathscr{M}_{8}=\frac{\alpha k}{(\alpha+k)(\alpha+2 k)}+\frac{i \alpha(1-\lambda)}{2 k(i+2)}+k B_{k}(s k+k, \alpha+k) \\
& -\frac{k}{\alpha+s k+2 k} \\
& \mathscr{M}_{9}=\frac{\alpha k}{(\alpha+k)(\alpha+2 k)}-k B_{k}(s k+2 k, \alpha+k)+\frac{i \alpha(1-\lambda)(i+3)}{2 k(i+1)(i+2)} \\
& +\frac{k^{2}}{(\alpha+s k+k)(\alpha+s k+2 k)}, \\
& \mathscr{M}_{10}=\frac{k^{2}}{(k \sigma+\alpha+2 k)(k \sigma+\alpha+3 k)} \\
& +\frac{\alpha}{k}(1-\lambda)\left(\frac{1}{(\sigma+2)(\sigma+3)}+k B_{k}(3 k, k \sigma+k)\right) \\
& -k B_{k}(k \sigma+2 k, \alpha+k)+k B_{k}(k \sigma+3 k, \alpha+k) \\
& +k B_{k}(\alpha+3 k, k \sigma+k)-k B_{k}(3 k, k \sigma+\alpha+k) \text {. }
\end{aligned}
$$

Proof. Using Lemma 10. improved power mean integral inequality and $\left|\mathscr{F}^{\prime}\right|^{q}$ is strongly $n$-polynomial preinvex function of higher order, we have

$$
\begin{aligned}
& \mid \frac{(k+\alpha(1-\lambda)) \mathscr{F}(a+\zeta(b, a))+(k-\alpha(1-\lambda)) \mathscr{F}(a)}{2 k} \\
& \quad-\frac{\Gamma_{k}(\alpha+k)}{2 \zeta^{a / k}(b, a)}\left({ }_{k} \widetilde{\mho}_{a^{+}}^{\alpha} \mathscr{F}(a+\zeta(b, a))+{ }_{k} \widetilde{\mho}_{(a+\zeta(b, a))^{\alpha}}^{\alpha} \mathscr{F}(a)\right) \mid \\
& =\frac{\zeta(b, a)}{2}\left|\int_{0}^{1}\left(t^{a / k}+\frac{\alpha}{k}(1-\lambda)-(1-t)^{a / k}\right) \mathscr{F}^{\prime}(a+t \zeta(b, a)) \mathrm{d} t\right| \\
& \leq \frac{\zeta(b, a)}{2}\left(\int_{0}^{1}(1-t)\left(t^{a / k}+\frac{\alpha}{k}(1-\lambda)-(1-t)^{a / k}\right) \mathrm{d} t\right)^{1-1 / q} \\
& \quad \times\left(\int_{0}^{1}(1-t)\left(t^{a / k}+\frac{\alpha}{k}(1-\lambda)-(1-t)^{a / k}\right)\left|\mathscr{F}^{\prime}(a+t \zeta(b, a))\right|^{q} \mathrm{~d} t\right)^{1 / q} \\
& \quad+\frac{\zeta(b, a)}{2}\left(\int_{0}^{1} t\left(t^{a / k}+\frac{\alpha}{k}(1-\lambda)-(1-t)^{a / k}\right) \mathrm{d} t\right)^{1-1 / q} \\
& \quad \times\left(\int_{0}^{1} t\left(t^{a / k}+\frac{\alpha}{k}(1-\lambda)-(1-t)^{a / k}\right)\left|\mathscr{F}^{\prime}(a+t \zeta(b, a))\right|^{q} \mathrm{~d} t\right)^{1 / q}
\end{aligned}
$$

$$
\begin{aligned}
\leq & \frac{\zeta(b, a)}{2}\left(\frac{\alpha}{2 k}(1-\lambda)-\frac{\alpha k}{(\alpha+k)(\alpha+2 k)}\right)^{1-1 / q} \\
& \times\left(\frac{\left|\mathscr{F}^{\prime}(a)\right|^{q}}{n} \sum_{i=1}^{n} \int_{0}^{1}(1-t)\left[1-t^{i}\right]\left(t^{a / k}+\frac{\alpha}{k}(1-\lambda)-(1-t)^{a / k}\right) \mathrm{d} t\right. \\
& +\frac{\left|\mathscr{F}^{\prime}(b)\right|^{q}}{n} \sum_{i=1}^{n} \int_{0}^{1}(1-t)\left[1-(1-t)^{i}\right]\left(t^{a / k}+\frac{\alpha}{k}(1-\lambda)-(1-t)^{a / k}\right) \mathrm{d} t \\
& -\mu\|\zeta(b, a)\|^{\sigma} \int_{0}^{1}(1-t)\left[t^{\sigma}(1-t)+t(1-t)^{\sigma}\right]\left(t^{a / k}+\frac{\alpha}{k}(1-\lambda)\right. \\
& \left.\left.-(1-t)^{a / k}\right) \mathrm{~d} t\right)^{1 / q}+\frac{\zeta(b, a)}{2}\left(\frac{\alpha k}{(\alpha+k)(\alpha+2 k)}+\frac{\alpha}{2 k}(1-\lambda)\right)^{1-1 / q} \\
& \times\left(\frac{\left|\mathscr{F}^{\prime}(a)\right|^{q}}{n} \sum_{i=1}^{n} \int_{0}^{1} t\left[1-t^{i}\right]\left(t^{a / k}+\frac{\alpha}{k}(1-\lambda)-(1-t)^{a / k}\right) \mathrm{d} t\right. \\
& +\frac{\left|\mathscr{F}^{\prime}(b)\right|^{q}}{n} \sum_{i=1}^{n} \int_{0}^{1} t\left[1-(1-t)^{i}\right]\left(t^{a / k}+\frac{\alpha}{k}(1-\lambda)-(1-t)^{a / k}\right) \mathrm{d} t \\
& \left.-\mu\|\zeta(b, a)\|^{\sigma} \int_{0}^{1} t\left[t^{\sigma}(1-t)+t(1-t)^{\sigma}\right]\left(t^{a / k}+\frac{\alpha}{k}(1-\lambda)-(1-t)^{a / k}\right) \mathrm{d} t\right)^{1 / q} \\
= & \frac{\zeta(b, a)}{2}\left(\frac{\alpha}{2 k}(1-\lambda)-\frac{\alpha k}{(\alpha+k)(\alpha+2 k)}\right)^{1-1 / q}\left(\frac{\left|\mathscr{F}^{\prime}(a)\right|^{q}}{n} \sum_{i=1}^{n} \mathscr{M}_{5}\right. \\
& \left.+\frac{\left|\mathscr{F}^{\prime}(b)\right|^{q}}{n} \sum_{i=1}^{n} \mathscr{M}_{6}-\mu\|\zeta(b, a)\|^{\sigma} \mathscr{M}_{7}\right)^{1 / q} \\
& \left.+\frac{\zeta(b, a)}{2}\left(\frac{\left.\alpha \mathscr{F}^{\prime}(b)\right|^{q}}{(\alpha+k)(\alpha+2 k)} \sum_{i=1}^{n} \mathscr{M}_{9}-\mu\|\zeta(b, a)\|^{\sigma} \mathscr{M}_{10}\right)^{1 / q} \cdot(1-\lambda)\right)^{1-1 / q}\left(\frac{\left|\mathscr{F}^{\prime}(a)\right|^{q}}{n} \sum_{i=1}^{n} \mathscr{M}_{8}\right. \\
& \alpha k
\end{aligned}
$$

This completes the proof.

\section{Conclusion}

In this article, we have introduced the notion of strongly $n$ -polynomial preinvex function of higher order. We have derived a new $k$-fractional analogue of classical HermiteHadamard's integral inequality utilizing the class of strongly $n$-polynomial preinvex functions. We established a new auxiliary result pertaining to $k$-fractional integrals, and utilizing this new result, we obtained several new variants of trapezium-like inequalities using the concept of strongly $n$ -polynomial preinvex functions of higher order. We would like to emphasize here that we can recapture some other new results from the main results of this article under some suitable conditions. For example, if we take $\sigma=2$, then all the results reduce to the results for strongly $n$-polynomial preinvex functions. If $\zeta(b, a)=b-a$, then we have results for $n$-polynomial convex functions of higher order. Similarly for other suitable choices, other new and known results, we left the details to interested readers. This shows that the results obtained in this article are quite a unifying one. We hope that the ideas and techniques of this article will inspire interested readers.

\section{Data Availability}

No data were used to support this study. 


\section{Conflicts of Interest}

The authors declare that there are no conflicts of interest regarding the publication of this paper.

\section{Authors' Contributions}

M. U. Awan gave Definition 8, carried out the proof of Theorem 9 and drafted the manuscript. S. Talib carried out the proof of Theorems 10 and 11. M. A. Noor carried out the proof of Theorem 12. Y.-M. Chu provided the main idea, carried out the proof of Theorem 13, completed the final revision, and submitted the article. K. I. Noor carried out the proof of Theorem 14. All authors read and approved the final manuscript.

\section{Acknowledgments}

This work was supported by the Natural Science Foundation of China (Grant No. 61673169). The authors are thankful to the editor and the anonymous referee for their valuable comments and suggestions.

\section{References}

[1] S. Rashid, M. A. Noor, K. I. Noor, F. Safdar, and Y.-M. Chu, "Hermite-Hadamard type inequalities for the class of convex functions on time scale," Mathematics, vol. 7, no. 10, p. 956, 2019.

[2] S. Zaheer Ullah, M. Adil Khan, and Y.-M. Chu, "A note on generalized convex functions," Journal of Inequalities and Applications, vol. 2019, no. 1, 2019.

[3] M. Adil Khan, M. Hanif, Z. A. Khan, K. Ahmad, and Y.M. Chu, "Association of Jensen's inequality for s-convex function with Csiszár divergence," Journal of Inequalities and Applications, vol. 2019, no. 1, 2019.

[4] M. A. Latif, S. Rashid, S. S. Dragomir, and Y.-M. Chu, "Hermite-Hadamard type inequalities for co-ordinated convex and qausi-convex functions and their applications," Journal of Inequalities and Applications, vol. 2019, no. 1, 2019.

[5] I. Abbas Baloch and Y.-M. Chu, "Petrović-type inequalities for harmonic $h$-convex functions," Journal of Function Spaces, vol. 2020, Article ID 3075390, 7 pages, 2020.

[6] M. U. Awan, N. Akhtar, A. Kashuri, M. A. Noor, and Y.M. Chu, " $2 D$ approximately reciprocal $\rho$-convex functions and associated integral inequalities," AIMS Mathematics, vol. 5, no. 5, pp. 4662-4680, 2020.

[7] M. U. Awan, N. Akhtar, S. Iftikhar, M. A. Noor, and Y.M. Chu, "New Hermite-Hadamard type inequalities for npolynomial harmonically convex functions," Journal of Inequalities and Applications, vol. 2020, no. 1, 2020.

[8] Y. Khurshid, M. Adil Khan, and Y.-M. Chu, "Conformable fractional integral inequalities for GG- and GA-convex functions," AIMS Mathematics, vol. 5, no. 5, pp. 5012-5030, 2020.

[9] Y. Khurshid, M. Adil Khan, and Y.-M. Chu, "Conformable integral version of Hermite-Hadamard-Fejér inequalities via $\eta$-convex functions," AIMS Mathematics, vol. 5, no. 5, pp. 5106-5120, 2020.

[10] S. Rashid, R. Ashraf, M. A. Noor, K. I. Noor, and Y.-M. Chu, "New weighted generalizations for differentiable exponentially convex mapping with application," AIMS Mathematics, vol. 5, no. 4, pp. 3525-3546, 2020.

[11] S. Rashid, İ. İşcan, D. Baleanu, and Y.-M. Chu, "Generation of new fractional inequalities via n polynomials s-type convexity with applications," Advances in Difference Equations, vol. 2020, no. 1, 2020.

[12] T.-H. Zhao, L. Shi, and Y.-M. Chu, "Convexity and concavity of the modified Bessel functions of the first kind with respect to Hölder means," Revista de la Real Academia de Ciencias Exactas, Físicas y Naturales Serie A Matemáticas, vol. 114, no. 2, article 96, p. 14, 2020.

[13] T. Abdeljawad, S. Rashid, H. Khan, and Y.-M. Chu, "On new fractional integral inequalities for p-convexity within interval-valued functions," Advances in Difference Equations, vol. 2020, no. 1, 2020.

[14] P. Agarwal, M. Kadakal, İ. İşcan, and Y.-M. Chu, "Better approaches for $n$-times differentiable convex functions," Mathematics, vol. 8, no. 6, p. 950, 2020.

[15] M.-K. Wang, H.-H. Chu, Y.-M. Li, and Y.-M. Chu, "Answers to three conjectures on convexity of three functions involving complete elliptic integrals of the first kind," Applicable Analysis and Discrete Mathematics, vol. 14, no. 1, pp. 255-271, 2020.

[16] M. A. Khan, J. Pečarić, and Y.-M. Chu, "Refinements of Jensen's and McShane's inequalities with applications," AIMS Mathematics, vol. 5, no. 5, pp. 4931-4945, 2020.

[17] S. Khan, M. Adil Khan, and Y.-M. Chu, "Converses of the Jensen inequality derived from the Green functions with applications in information theory," Mathematicsl Methods in the Applied Sciences, vol. 43, no. 5, pp. 2577-2587, 2020.

[18] W.-M. Qian, W. Zhang, and Y.-M. Chu, "Bounding the convex combination of arithmetic and integral means in terms of oneparameter harmonic and geometric means," Miskolc Mathematical Notes, vol. 20, no. 2, pp. 1157-1166, 2019.

[19] S. Rafeeq, H. Kalsoom, S. Hussain, S. Rashid, and Y.-M. Chu, "Delay dynamic double integral inequalities on time scales with applications," Advances in Difference Equations, vol. 2020, no. 1, 2020.

[20] S. Rashid, F. Jarad, and Y.-M. Chu, "A note on reverse Minkowski inequality via generalized proportional fractional integral operator with respect to another function," Mathematical Problems in Engineering, vol. 2020, Article ID 7630260, 12 pages, 2020.

[21] S. Rashid, F. Jarad, H. Kalsoom, and Y.-M. Chu, "On PólyaSzegö and Čebyšev type inequalities via generalized kfractional integrals," Advances in Difference Equations, vol. 2020, no. 1, 2020.

[22] S. Rashid, F. Jarad, M. A. Noor, H. Kalsoom, and Y.-M. Chu, "Inequalities by means of generalized proportional fractional integral operators with respect to another function," Mathematics, vol. 7, no. 12, article 1225, p. 18, 2019.

[23] M.-K. Wang, Z.-Y. He, and Y.-M. Chu, "Sharp power mean inequalities for the generalized elliptic integral of the first kind," Computational Methods and Function Theory, vol. 20, no. 1, pp. 111-124, 2020.

[24] M.-K. Wang, M.-Y. Hong, Y.-F. Xu, Z.-H. Shen, and Y.$\mathrm{M}$. Chu, "Inequalities for generalized trigonometric and hyperbolic functions with one parameter," Journal of Mathematical Inequalities, vol. 14, no. 1, pp. 1-21, 2020.

[25] Z.-H. Yang, W.-M. Qian, W. Zhang, and Y.-M. Chu, "Notes on the complete elliptic integral of the first kind," Mathematical Inequalities \& Applications, vol. 23, no. 1, pp. 77-93, 2020. 
[26] T.-H. Zhao, M.-K. Wang, and Y.-M. Chu, "A sharp double inequality involving generalized complete elliptic integral of the first kind," AIMS Mathematics, vol. 5, no. 5, pp. 45124528, 2020.

[27] S.-S. Zhou, S. Rashid, F. Jarad, H. Kalsoom, and Y.-M. Chu, "New estimates considering the generalized proportional Hadamard fractional integral operators," Advances in Difference Equations, vol. 2020, no. 1, 2020.

[28] S. Rashid, Z. Hammouch, F. Jarad, and Y.-M. Chu, "New estimates of integral inequalities via generalized proportional fractional integral operator with respect to another function," Fractals, vol. 28, no. 8, article 2040027, p. 12, 2020.

[29] S. Rashid, Z. Hammouch, D. Baleanu, and Y.-M. Chu, "New generalizations in the sense of the weighted non-singular fractional integral operator," Fractals, vol. 28, no. 7, article 2040003, p. 11, 2020

[30] S. Hussain, J. Khalid, and Y.-M. Chu, "Some generalized fractional integral Simpson's type inequalities with applications," AIMS Mathematics, vol. 5, no. 6, pp. 5859-5883, 2020.

[31] L. Xu, Y.-M. Chu, S. Rashid, A. A. El-Deeb, and K. S. Nisar, "On new unified bounds for a family of functions via fractional q-calculus theory," Journal of Function Spaces, vol. 2020, Article ID 4984612, 9 pages, 2020.

[32] S. Rashid, A. Khalid, G. Rahman, K. S. Nisar, and Y.-M. Chu, "On new modifications governed by quantum Hahn's integral operator pertaining to fractional calculus," Journal of Function Spaces, vol. 2020, Article ID 8262860, 12 pages, 2020.

[33] M. A. Hanson, "On sufficiency of the Kuhn-Tucker conditions," Journal of Mathematical Analysis and Applications, vol. 80, no. 2, pp. 545-550, 1981.

[34] B. D. Craven, "Duality for generalized convex fractional programs," in Generalized Convexity in Optimization and Economics, S. Schaible and W. T. Ziemba, Eds., pp. 473-489, Academic Press, New York, NY, USA, 1981.

[35] S. Mititelu, "Invex sets," Studii și Cercetări Matematice, vol. 46, no. 5, pp. 529-532, 1994.

[36] T. Weir and B. Mond, "Pre-invex functions in multiple objective optimization," Journal of Mathematical Analysis and Applications, vol. 136, no. 1, pp. 29-38, 1988.

[37] M. A. Noor, K. I. Noor, M. U. Awan, and J.-Y. Li, "On Hermite-Hadamard inequalities for $h$-preinvex functions," Filomat, vol. 28, no. 7, pp. 1463-1474, 2014.

[38] T. Toplu, M. Kadakal, and İ. İşcan, "On $n$-polynomial convexity and some related inequalities," AIMS Mathematics, vol. 5, no. 2, pp. 1304-1318, 2020.

[39] S. Karamardian, "The nonlinear complementarity problem with applications II," J. Optim. Theory Appl., vol. 4, no. 3, pp. 167-181, 1969.

[40] B. T. Poljak, "Existence theorems and convergence of minimizing sequences for extremal problems with constraints," Doklady Akademii Nauk SSSR, vol. 166, pp. 287-290, 1966.

[41] G. H. Lin and M. Fukushima, "Some exact penalty results for nonlinear programs and mathematical programs with equilibrium constraints," Journal of Optimization Theory and Applications, vol. 118, no. 1, pp. 67-80, 2003.

[42] M. Adil Khan, A. Iqbal, M. Suleman, and Y.-M. Chu, "Hermite-Hadamard type inequalities for fractional integrals via Green's function," Journal of Inequalities and Applications, vol. 2018, no. 1, 2018.

[43] M. Adil Khan, N. Mohammad, E. R. Nwaeze, and Y.-M. Chu, "Quantum Hermite-Hadamard inequality by means of a
Green function," Advances in Difference Equations, vol. 2020, no. 1,2020

[44] M. U. Awan, S. Talib, Y.-M. Chu, M. A. Noor, and K. I. Noor, "Some new refinements of Hermite-Hadamard-type inequalities involving $\psi_{k}$-Riemann-Liouville fractional integrals and applications," Mathematical Problems in Engineering, vol. 2020, Article ID 3051920, 10 pages, 2020.

[45] A. Iqbal, M. Adil Khan, S. Ullah, and Y.-M. Chu, "Some new Hermite-Hadamard-type inequalities associated with conformable fractional integrals and their applications," Journal of Function Spaces, vol. 2020, Article ID 9845407, 18 pages, 2020.

[46] A. A. Kilbas, H. M. Srivastava, and J. J. Trujillo, Theory and Applications of Fractional Differential Equations, Elsevier Science B.V., Amsterdam, 2006.

[47] M. Z. Sarikaya, E. Set, H. Yaldiz, and N. Basak, "HermiteHadamard's inequalities for fractional integrals and related fractional inequalities," Mathematical and Computer Modelling, vol. 57, no. 9-10, pp. 2403-2407, 2013.

[48] K. S. Nisar, G. Rahman, A. Khan, A. Tassaddiq, and M. S. Abouzaid, "Certain generalized fractional integral inequalities," AIMS Mathematics, vol. 5, no. 2, pp. 1588-1602, 2020.

[49] G. Rahman, K. S. Nisar, A. Ghaffar, and F. Qi, "Some inequalities of the Grüss type for conformable $k$-fractional integral operators," Revista de la Real Academia de Ciencias Exactas, Físicas y Naturales Serie A Matemáticas, vol. 114, no. 1, p. 9, 2020.

[50] A. Tassaddiq, G. Rahman, K. S. Nisar, and M. Samraiz, "Certain fractional conformable inequalities for the weighted and the extended Chebyshev functionals," Advances in Difference Equations, vol. 2020, no. 1, 2020.

[51] R. Daz and E. Pariguan, "On hypergeometric functions and Pochhammer k-symbol,” Divulgaciones Matematicas, vol. 15, no. 2, pp. 179-192, 2007.

[52] M. Z. Sarikaya and A. Karaca, "On the $k$-Riemann-Liouville fractional integral and applications," International Journal of Statistics and Mathematics, vol. 1, no. 3, pp. 33-43, 2014.

[53] C.-J. Huang, G. Rahman, K. S. Nisar, A. Ghaffar, and F. Qi, "Some inequalities of the Hermite-Hadamard type for $k$-fractional conformable integrals," Australian Journal of Mathematical Analysis and Applications, vol. 16, no. 1, p. 7, 2019.

[54] G. Rahman, K. S. Nisar, T. Abdeljawad, and S. Ullah, "Certain fractional proportional integral inequalities via convex functions," Mathematics, vol. 8, no. 2, p. 222, 2020.

[55] S. R. Mohan and S. K. Neogy, "On invex sets and preinvex functions," Journal of Mathematical Analysis and Applications, vol. 189, no. 3, pp. 901-908, 1995. 